\title{
A Guide for applying a revised version of the PARIHS framework for implementation
}

\author{
Cheryl B Stetler ${ }^{1,2^{*}}$, Laura J Damschroder ${ }^{3}$, Christian D Helfrich ${ }^{4,5}$ and Hildi J Hagedorn ${ }^{6,7}$
}

\begin{abstract}
Background: Based on a critical synthesis of literature on use of the Promoting Action on Research Implementation in Health Services (PARIHS) framework, revisions and a companion Guide were developed by a group of researchers independent of the original PARIHS team. The purpose of the Guide is to enhance and optimize efforts of researchers using PARIHS in implementation trials and evaluations.

Methods: Authors used a planned, structured process to organize and synthesize critiques, discussions, and potential recommendations for refinements of the PARIHS framework arising from a systematic review. Using a templated form, each author independently recorded key components for each reviewed paper; that is, study definitions, perceived strengths/limitations of PARIHS, other observations regarding key issues and recommendations regarding needed refinements. After reaching consensus on these key components, the authors summarized the information and developed the Guide.
\end{abstract}

Results: A number of revisions, perceived as consistent with the PARIHS framework's general nature and intent, are proposed. The related Guide is composed of a set of reference tools, provided in Additional files. Its core content is built upon the basic elements of PARIHS and current implementation science.

Conclusions: We invite researchers using PARIHS for targeted evidence-based practice (EBP) implementations with a strong task-orientation to use this Guide as a companion and to apply the revised framework prospectively and comprehensively. Researchers also are encouraged to evaluate its use relative to perceived strengths and issues. Such evaluations and critical reflections regarding PARIHS and our Guide could thereby promote the framework's continued evolution.

\section{Background}

In October 2010, a critical synthesis of literature on the use of the Promoting Action on Research Implementation in Health Services (PARIHS) framework was published in Implementation Science [1]. PARIHS is a widely cited conceptual framework that conceives of three key, interacting elements that influence successful implementation of evidence-based practices (EBPs): Evidence $(E)$, Context $(C)$, and Facilitation $(F)$. The literature synthesis identified key strengths and issues as regards the framework.

A subgroup of the synthesis authors drew upon the above results to revise PARIHS for use by researchers in the Veteran's Health Administration (VA); that is, in trials or evaluations focused on implementation of

\footnotetext{
* Correspondence: cheryl.stetler@comcast.net

${ }^{1}$ Independent Consultant, Amherst, Massachusetts, USA

Full list of author information is available at the end of the article
}

targeted EBPs. A companion document, or Guide, also was developed to provide direction on how this revised version could be operationalized. Together, the framework modifications and Guide addressed barriers to the use of PARIHS previously encountered by VA researchers, in part due to the framework's limitations [1]. It is important to note that although we propose a number of revisions and comment on how best to use PARIHS, we have built on the original work of the PARIHS team [2-5]; and while we have shared our work with members of that team, this version of PARIHS and our related Guide were developed independently. It does not necessarily reflect the PARIHS team's views. This work further reflects our efforts to operationalize the PARIHS framework based on our VA research context, our VA experience with PARIHS, and our critical review [1]. Were others to follow the same process, they might come to different interpretations and conclusions.

\section{Biomed Central}

(c) 2011 Stetler et al; licensee BioMed Central Ltd. This is an Open Access article distributed under the terms of the Creative Commons Attribution License (http://creativecommons.org/licenses/by/2.0), which permits unrestricted use, distribution, and reproduction in any medium, provided the original work is properly cited. 
Our Guide is intended to enhance and optimize the efforts of those choosing to use PARIHS as their theoretical framework. It is designed to enable users to more clearly and consistently define and apply relevant terms. Further, it is designed to facilitate diagnostic analysis of framework elements, selection of an appropriate implementation strategy, and measurement of Successful Implementation. It is hoped that similar syntheses and guides will be developed for other implementation theories, models, and frameworks [6]. Within the VA, where no single theory takes precedence over any other, efforts are underway to enhance operationalization of other frameworks and models by mapping their elements to constructs identified through a Consolidated Framework for Implementation Research (CFIR)[7].

Since the intent of this paper is to provide others interested in using PARIHS with tool-based, practical guidance, we rely heavily on additional files. These files equip users of the framework with the following: a set of definitions for elements/sub-elements, tips in the form of observations about use of elements/subelements, and a set of questions for diagnostic analysis and planning. All of the separate components of the actual Guide are contained in additional files (see Additional Files 1, 2, 3 and 4). The main narrative provides only overview information and pointers regarding various Guide components. Specifically, this overview briefly describes the basic underlying PARIHS framework [2-5], its limitations and related issues [1], the structured process and frames of reference used to identify modifications and create the Guide, and the revisions to the original framework [2-5]. It also provides sample material from additional files to give readers a better feel for their content and potential usefulness.

\section{Brief overview of PARIHS}

PARIHS can be characterized as an impact or explanatory framework [6], originally developed in 1998 [8] and refined over time based on concept analyses and exploratory research $[2-5,9,10]$.

Before using our Guide, it is important that users be familiar with the underlying framework of PARIHS [2,3,5] (e.g., see Rycroft-Malone et al. [3] for a recent depiction of the framework, including its key sub-elements and explanatory material; also see Kitson et al.'s discussion regarding theoretical issues in general and PARIHS' status specifically, noting the potential diagnostic and evaluative questions they provide in a related appendix [5]). Another, more recent publication provides an overview of the framework, its underlying assumptions, developmental work, and its use by others [11]. Key aspects of the PARIHS framework are herein summarized in Table 1. Figure 1 outlines the sub- elements of each of the core elements, as described in the PARIHS team's 2004 refinement [3].

In summary, PARIHS can be selected as a broad framework to guide development of a program of implementation interventions that effectively enable EBPrelated changes. Specifically, it can be used to diagnose critical elements related to implementation of an EBP ( $E$ and $C$ ) and thence development of an implementation strategy $(F)$ to enable successful and sustained change. A PARIHS-based diagnostic analysis can additionally engage stakeholders in self-reflection regarding critical aspects of implementation and the related nature of needed change [12].

\section{PARIHS limitations and related issues}

Strengths of the PARIHS framework identified through our published synthesis included the following: its intuitive appeal, provision of a basic "to-do" list, flexibility in application, and inclusion of Successful Implementation as the desired outcome [1]. Of particular importance to development of the Guide were its identified limitations and related issues [1]. These included the following, which are further described in Table 2:

- Lack of conceptual clarity, specificity, and transparency, which results in different interpretations of PARIHS concepts by different researchers

- Lack of inclusion of relevant elements perceived to be critical to implementation and congruent with the main intent of PARIHS

- Lack of well-developed instrumentation and evaluation measures, as well as limited evaluation of actual use or perceived usefulness of the framework.

No published studies were identified that used the framework comprehensively and prospectively to develop an implementation project. The ability to fully evaluate its usefulness thus has been limited.

\section{Methods}

Revising PARIHS for use in task-oriented implementation Our objective in developing the Guide was to meet the needs of VA researchers interested in understanding the nuts and bolts of operationalizing PARIHS. More specifically, our objective was two-fold: (1) provide guidance on how best to apply/operationalize the framework within QUERI's [Quality Enhancement Research Initiative] action-oriented approach [13-15] and (2) enable more effective use of the framework by addressing identified barriers (Table 2). (Note: Italicized sentences here and in the next section come from our internal PARIHS synthesis/application project plan.)

Given this practical need, after completion of the synthesis groundwork, the authors used a planned, 


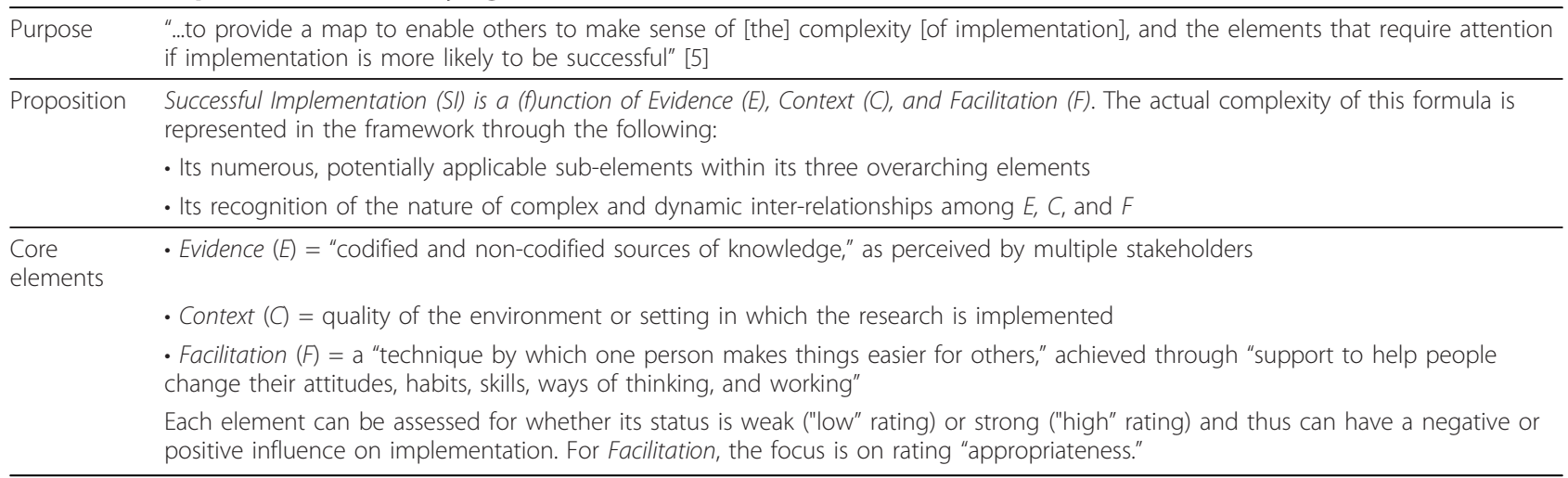

PARIHS = Promoting Action on Research Implementation in Health Services.

structured process to organize and bring together into a coherent whole the substance of our critiques, related discussions, and potential recommendations for refinements/adaptations of the PARIHS framework-for use within the context of QUERI-like implementation projects. Specifically, the authors did the following:

1) Utilizing finalized critiques from the published synthesis [1], each author independently recorded key components for each reviewed paper on a templated form. This form focused on the study's definition of elements, perceived strengths/limitations of PARIHS highlighted by the study, other observations regarding key PARIHS issues, and recommendations regarding refinements consistent with the intent of the basic framework ... in light of the QUERI framework, QUERI experience and current science.

2) Each author independently reviewed selected components of two other published syntheses that analyzed the concept of Context $[7,16]$.

3) As a group, the authors critically reviewed, discussed, and themed the above information at a two-day intensive face-to-face meeting.)

4) As a group, the authors reached consensus on the above key components, including the clarity/lack of clarity of language found in various definitions, and then identified opportunities to improve the framework.

Information from step 4 was used to draft a Guide. Critical to this draft was the original PARIHS framework, primarily its two most recent versions $[2,3]$ and the 2008 paper and Appendix [5]. Feedback was obtained from VA implementation researchers [1] and others familiar with PARIHS, and minor refinements made.

Critical to understanding the general implementation approach embedded within this Guide is the nature of QUERI's action-oriented paradigm. This implementation/research paradigm served as an implicit background or frame of reference for overall author deliberations. It distinguishes two general types of implementation situations and emphasizes a set of innovative concepts.

\section{Types of implementation}

We distinguish two general types of implementation situations:

- one with a task-oriented purpose, where a specific intervention is being implemented within a relatively short timeframe (such as implementing a new procedure or care process)

- one with a broader "organizational" purpose, where implementation strategies are targeted at transformational change within one or more levels of an institution (such as changing culture to be more receptive to using EBPs on a routine basis [17]).

The primary focus of QUERI projects, and thus the purpose of this Guide, is to assist with more short-term, targeted EBP implementation studies with a strong task orientation $[14,15]$. We highlight this distinction because it influenced how we approached framework refinements and identified observations/tips in the reference tools.

In short-term, task-oriented situations, implementation efforts are unlikely to target broad changes in the multiple sub-elements related to culture, evaluation, or leadership. We therefore focused on defining and highlighting only those aspects of PARIHS elements that might realistically be modified in a relatively short period of time.

It is important to further distinguish our use of the terms task versus organizational purpose from the PARIHS framework's approach to Facilitation. The latter envisages the purpose of Facilitation to occur along a continuum from primarily "task" to "holistic." The former focuses on "a 'doing for others' role ... [and is] more discrete, practical, technical and task driven," while the latter focuses on "an "enabling and empowering' role which is more developmental" [5]. In most cases, task-oriented EBP implementation situations will 


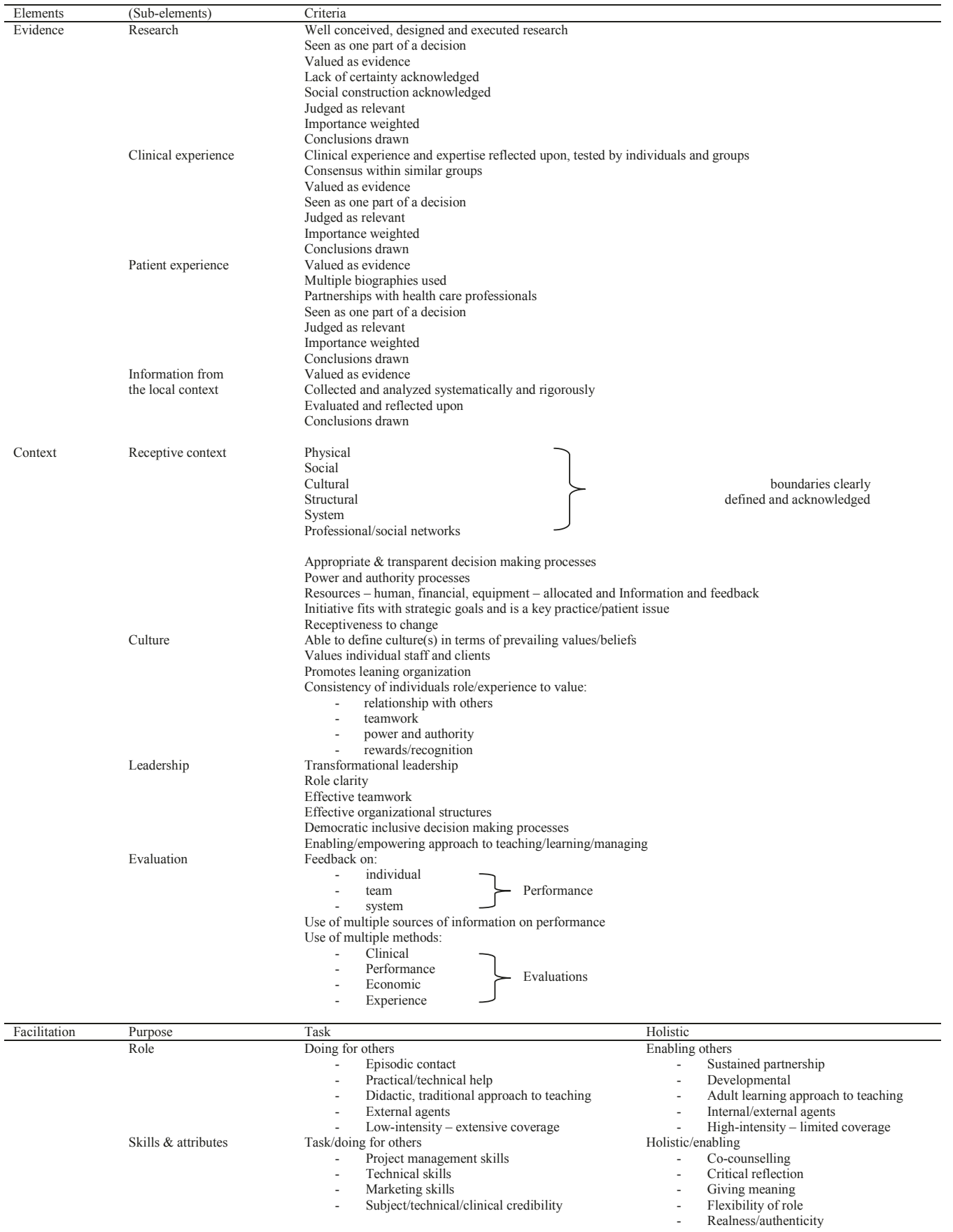

Figure 1 Key elements for implementing evidence into practice [3]. This figure reproduces the PARIHS team's 2004 version of its framework, with all its elements and sub-elements and "criteria," from the following publication: Rycroft-Malone J, Harvey G, Seers K, Kitson A, McCormack B, Titchen A: An exploration of the factors that influence the implementation of evidence into practice. J Clin Nurs, 2004, 13(8): 913-924. It is reproduced with permission. "Criteria" highlight the conditions more likely needed for, or critical to, successful implementation.

rely more heavily on task-focused or "mixed" Facilitation methods; on the other hand, transformational initiatives that have an organizational redesign goal will rely more heavily on holistic Facilitation [5].

\section{Innovative, action-oriented QUERI concepts}

As QUERI developed over time, a set of concepts guided its implementation research activities. Some of these concepts relate to QUERI innovations or contributions 


\begin{tabular}{|c|c|}
\hline \multirow[t]{3}{*}{ Conceptual clarity } & $\begin{array}{l}\text { - Ambiguity in certain terms and phrases; for example, when assessing Evidence, one criterion for } \\
\text { "high" research evidence is that "social construction [is] acknowledged." Cross-country and } \\
\text { philosophical differences may contribute to this perception of "obscurity" in such language. }\end{array}$ \\
\hline & $\begin{array}{l}\text { - Lack of specificity in element/sub-element names and definitions, making it unclear what is actually } \\
\text { included/excluded; for example, one of the elements is titled Context, as is one of its sub-elements, } \\
\text { Receptive Context. }\end{array}$ \\
\hline & $\begin{array}{l}\text { - Lack of transparency or specificity in how to operationalize various sub-elements, such as clinical } \\
\text { experience or patient experience. }\end{array}$ \\
\hline \multirow[t]{5}{*}{ "Missing" components } & - Lack of a definition for Successful Implementation (SI). \\
\hline & $\begin{array}{l}\text { - Need to explicitly designate motivation for change/importance of a "recognized need for change" } \\
\text { [34], as pointed out by Ellis et al. }\end{array}$ \\
\hline & $\begin{array}{l}\text { - Potential value of making more explicit a critical set of innovation attributes (e.g., per Rogers' } \\
\text { diffusion of innovation theory [33]). }\end{array}$ \\
\hline & $\begin{array}{l}\text { - Removal of clearly stated attributes of a facilitator after earliest version of PARIHS (i.e., general } \\
\text { credibility, authenticity, and respect). }\end{array}$ \\
\hline & $\begin{array}{l}\text { - Insufficient guidance or clarification under Facilitation regarding the task of developing needed } \\
\text { "change...strategies" [5], based on suggested diagnostic analysis of } E \text { and } C \text {-and lack of inclusion of } \\
\text { common implementation interventions that a Facilitator employs, reinforces, or proposes to enhance } \\
\text { adoption. }\end{array}$ \\
\hline \multirow[t]{2}{*}{$\begin{array}{l}\text { Under-developed evaluation and related } \\
\text { instrumentation/measures }\end{array}$} & $\begin{array}{l}\text { - Few well-developed PARIHS-related instruments or other evaluative approaches to identify related } \\
\text { barriers/facilitators during diagnostic analysis or to evaluate successful implementation. }\end{array}$ \\
\hline & - Limited evaluation or means for evaluation of the theory's use/usefulness. \\
\hline
\end{tabular}

PARIHS = Promoting Action on Research Implementation in Health Services.

[7,14,15,17-24], others to the Stetler model of EBP $[25,26]$, and yet others to the general implementation science literature spanning the last decade [16,27-33]. Such concepts include, for example, strength of evidence, theoretical underpinnings, attributes of innovations, appropriate variation and qualifiers for use of evidence, social marketing and other recognized implementation interventions, sustainability, cost considerations for implementation, and critical leadership behaviors. Such concepts were familiar to the authors, were implicitly part of our decision-making, and ultimately influenced our development of the Guide's content in general and construction of the files' "Related Observations/Tips" most specifically.

\section{Results}

\section{Revisions to PARIHS}

Based on the above process and frames of reference, a number of modifications were made to the original PARIHS framework. Emphasis was placed on modifiable sub-elements or ones that might be buffered to reduce negative influences. This revised version of PARIHS is outlined in Table 3. Of particular note are the following:

- Changes were made both to wording and ordering of a few elements/sub-elements, as can be seen in comparing Table 3 to Figure 1. For example, the name of the Context element was amended (Contextual Readiness for Targeted EBP Implementation) to clearly indicate our task-oriented focus; and Leadership became the first sub-element under Context, indicating its prime importance in implementation. Nonetheless, it is important to note that the original PARIHS sub-elements of transformational leadership are still reflected within the Guide (e.g., role clarity and effective teamwork).

- A few items were added to core elements to reflect relevant features critical to implementation but missing from the framework (Table 2); for example, EBP Characteristics within Evidence now highlights attributes of an implementable form of "evidence" (i.e., the full form of an "EBP" innovation, such as a policy, procedure, or program). These additions were drawn from Roger's diffusion of innovation work [33] and the CFIR [7]. Some of these additions were already implicit within other Evidence sub-elements. As a result there may appear to be some overlap. However, these attributes were considered important enough to be expanded and made explicit, thus ensuring their consideration. This is particularly important because implementation decisions flow first from the nature of the implementable form of the Evidence and its characteristics.

Additionally, for Facilitation, implementation interventions beyond that of a facilitator role were inserted. This modification speaks in part to the 2008 PARIHS paper's comment regarding development of a "programme of change," that is, "task based, planned change programme approaches that meet the individual and team's learning needs...." [5]-and, we would add, that meet contextual needs identified through diagnostic analysis. As these programmes of change are likely to require "a range of different techniques" [5], we now make such 
Table 3 Revised PARIHS framework for a task-oriented approach to implementation: $S I=$ function of $E, C, F$

\begin{tabular}{|c|c|}
\hline Elements & Sub-elements \\
\hline \multirow[t]{12}{*}{ E: Evidence and EBP Characteristics } & - Research and published guidelines \\
\hline & - Clinical experiences and perceptions \\
\hline & - Patient experiences, needs, and preferences \\
\hline & - Local practice information \\
\hline & - Characteristics of the targeted EBP: \\
\hline & - Relative advantage \\
\hline & - Observability \\
\hline & - Compatibility \\
\hline & - Complexity \\
\hline & - Trialability \\
\hline & - Design quality and packaging \\
\hline & - Costs \\
\hline \multirow{4}{*}{$\begin{array}{l}\text { C: Contextual Readiness for Targeted EBP } \\
\text { Implementation }\end{array}$} & - Leadership support \\
\hline & - Culture \\
\hline & - Evaluation capabilities \\
\hline & - Receptivity to the targeted innovation/change \\
\hline \multirow[t]{8}{*}{ F: Facilitation } & Role of facilitator: \\
\hline & - Purpose, external and/or internal role \\
\hline & - Expectations and activities \\
\hline & - Skills and attributes of facilitator \\
\hline & $\begin{array}{l}\text { Other implementation interventions suggested per site diagnostic assessment } \\
\text { or relevant sources (e.g., prior research/literature and supplementary theories) } \\
\text { and used by the Facilitator and others }\end{array}$ \\
\hline & - Related to $E$ \\
\hline & - Related to C \\
\hline & - Other \\
\hline \multirow[t]{3}{*}{ SI: Successful Implementation } & - Implementation plan and its realization \\
\hline & $\begin{array}{l}\text { - EBP innovation uptake: uptake of clinical interventions and/or delivery system } \\
\text { interventions }\end{array}$ \\
\hline & - Patient and organizational outcomes achievement \\
\hline
\end{tabular}

PARIHS = Promoting Action on Research Implementation in Health Services; EBP = evidence-based practice.

techniques more explicit. This ties "Facilitation as an intervention" [5] to implementation interventions in general, which facilitators and others employ to enhance adoption.

- Successful Implementation is now visualized as an explicit part of the revised PARIHS "figure" (Table 3), with detailed definitions provided in the Guide (Additional File 4). This first effort at explicating the meaning of Successful Implementation is only preliminary and will benefit from ongoing attempts to operationalize it.

Finally, based on our synthesis, our frames of reference, and our framework modifications, we were able to construct a Guide (Table 4). Again, its intent is to enhance and optimize efforts of those using PARIHS as their theoretical framework. Within the Guide, the team used active, pragmatic language for each element/subelement-and, again, tied these changes to the original PARIHS framework material and its perceived intent.
Such language focuses on recognizable, measurable behaviors and minimizes what to us was abstract language less familiar to our researchers. The content of all additional files provides the following:

- Conceptual and operational definitions: This includes refined meanings of constructs within the framework, reflecting the team's interpretation of each element and related sub-element. These definitions are intended to facilitate in-depth understanding of each concept, guide application of the various elements, and identify potential questions for diagnostic analysis and planning.

- Observations and tips: This additional information, from the implementation literature and authors' experiences, is designed to enhance researchers' nuanced understanding of PARIHS elements/sub-elements. Tips also may facilitate design decisions.

As stated previously, the material contained across the additional files (i.e., the revised PARIHS Guide) is the 
Table 4 Additional files: Guide for applying a revised version of the PARIHS framework for implementation

\begin{tabular}{l}
\hline $\begin{array}{l}\text { A. Additional File 1: "EVIDENCE" Element: Evidence and EBP Characteristics } \\
(E)\end{array}$ \\
\hline - E element and related sub-elements \\
- Conceptual definitions \\
- Detailed observations/tips regarding sub-elements and \\
measurement \\
- Sample, optional questions to guide formative evaluation \\
\hline B. Additional File 2: "CONTEXT" Element: Contextual Readiness for \\
Targeted EBP Implementation (C) \\
- C element and related sub-elements \\
- Conceptual definitions \\
- Detailed observations/tips regarding sub-elements and \\
measurement \\
- Sample, optional questions to guide formative evaluation \\
\hline C. Additional File 3: "FACILITATION" (F) Element \\
- F element and related sub-elements \\
- Conceptual definitions \\
- Detailed observations/tips regarding sub-elements and \\
measurement \\
- Sample, optional questions to guide the team's project planning \\
\hline D. Additional File 4: "SUCCESSFUL IMPLEMENTATION" (SI) Element \\
- SI sub-elements \\
- Conceptual definitions \\
- Detailed observations/tips regarding sub-elements and \\
measurement \\
- Sample, optional questions to guide the team's development of \\
an evaluation plan
\end{tabular}

PARIHS = Promoting Action on Research Implementation in Health Services; $\mathrm{EBP}=$ evidence-based practice.

meat of this publication. It is intended to be used as an active reference tool for planning implementation research and evaluation. Tables 5, 6 and 7 provide the reader with a preview of these reference tools. Table 5 points out how we describe the potential use of an individual tool; Table 6 illustrates our approach to defining each of the core elements; and Table 7 demonstrates how an individual sub-element is presented in terms of its definitions, tips on use, and measurement.

\section{Summary and conclusions}

Based on a systematic, structured process, the authors have revised PARIHS and provided a detailed reference Guide to help researchers apply this framework. When using the Guide, readers should keep the following points in mind:

- The Guide relies on basic elements of PARIHS, as well as updates provided in Kitson and colleagues' 2008 paper and its appendix, specifically its diagnostic approach [5].

- A key revision objective was to minimize the original framework's limitations and related issues (Table 2).

- Our modifications are consistent with the general nature and intent of the PARIHS framework.

- Basic expectations for applying any framework, theory, or model were a guiding influence, that is, the need for clear conceptual and operational definitions, measurement approaches, and additional practical information about the realities of application.

- QUERI frames of reference and concepts affected development of Guide content, as did supplemental information from complementary theories such as Rogers, the Stetler model of EBP, and other selected concepts from implementation science. Modifications are thus responsive to the PARIHS team's suggestion [5] to draw on other theoretical perspectives; for example, "What theories would inform the way evidence has been conceptualized within the PARIHS framework?"

- The implementation knowledge and experiencebased lessons of the author team (published implementation scientists in the VA) influenced consensual judgments underlying the Guide.

- Our addition of "other implementation interventions" to the Facilitation element draws, in part, from a QUERI evaluation on facilitation wherein data suggested

Table 5 Illustration of Guide content: description of potential uses of a sample tool

\begin{tabular}{|c|c|}
\hline Element & Reference tool content \\
\hline $\begin{array}{l}\text { C: Contextual Readiness for Targeted EBP } \\
\text { Implementation }\end{array}$ & $\begin{array}{l}\text { Information in this and the other tools in this Revised PARIHS Guide can be used to prepare a proposal, } \\
\text { including related methodology, and follow-up reports. More specifically, this Context tool can be used to: }\end{array}$ \\
\hline - Leadership support & $\begin{array}{l}\text { - Think more specifically about the nature of Context and enhance communication of that } \\
\text { understanding to reviewers and other readers. }\end{array}$ \\
\hline - Culture & $\begin{array}{l}\text { - Identify potential Contextual barriers that may need to be better understood and/or addressed in the } \\
\text { implementation strategy (e.g., thinking through the type of leadership support that will be needed } \\
\text { given the type of innovation to be implemented). }\end{array}$ \\
\hline - Evaluation capabilities & $\begin{array}{l}\text { - Identify diagnostic/evaluative questions for a semi-structured interview relevant to the need to } \\
\text { understand selected aspects of the Context, applicable to this specific EBP change. }\end{array}$ \\
\hline \multirow[t]{2}{*}{$\begin{array}{l}\text { - Receptivity to the targeted } \\
\text { innovation/change }\end{array}$} & $\begin{array}{l}\text { - Develop and organize a retrospective interpretive evaluation [20] to explore the perceived influence } \\
\text { of Contextual features on implementation of the targeted EBP. }\end{array}$ \\
\hline & $\begin{array}{l}\text { NOTE: In all cases, the list of multiple items should be considered an optional menu from which to choose } \\
\text { components of prime relevance to implementation of the targeted EBP. }\end{array}$ \\
\hline
\end{tabular}

PARIHS = Promoting Action on Research Implementation in Health Services; EBP = evidence-based practice. 
Table 6 Illustration of Guide content: description of a core element

\begin{tabular}{|c|c|c|c|}
\hline Element & Conceptual definitions & Related observations/tips & Measurement \\
\hline \multirow[t]{3}{*}{$\begin{array}{l}\text { Evidence \& } \\
\text { EBP } \\
\text { Characteristics }\end{array}$} & $\begin{array}{l}\text { Evidence = Specified sources of } \\
\text { information relevant to a specific EBP, } \\
\text { including research/published guidelines, } \\
\text { clinical experience, patient experience, } \\
\text { and/or local practice information. }\end{array}$ & $\begin{array}{l}\text { As "evidence" is socially constructed [4], } \\
\text { the perceptions of targeted stakeholders } \\
\text { regarding the nature and quality of these } \\
\text { varying sources of evidence are key to } \\
\text { development of an implementation } \\
\text { strategy. }\end{array}$ & $\begin{array}{l}\text { Two quantitative measurement } \\
\text { instruments have been developed that } \\
\text { incorporate major components of PARIHS } \\
\text { related to Evidence: ORCA [18] and a survey } \\
\text { developed by Bahtsevani and colleagues } \\
\text { [35]. }\end{array}$ \\
\hline & $\begin{array}{l}\text { - These sources have presumably been } \\
\text { subjected to scrutiny (e.g., by the } \\
\text { research team or a national body) and } \\
\text { are judged to support or refute } \\
\text { effectiveness of a targeted EBP } \\
\text { intervention/recommendation. }\end{array}$ & $\begin{array}{l}\text { - This includes perception of the form } \\
\text { of the evidence-based clinical } \\
\text { recommendation/intervention (i.e., the } \\
\text { recommended practice as a guideline, } \\
\text { policy, procedure, protocol, program, } \\
\text { optional or forced function clinical } \\
\text { reminder, decision algorithm, etc.). At } \\
\text { times such transformed findings/ } \\
\text { "evidence" is supplemented with } \\
\text { additional content based on the } \\
\text { judgment or consensus of its creator } \\
\text { (e.g., consider the mixed nature of } \\
\text { various guidelines or protocols). }\end{array}$ & $\begin{array}{l}\text { Sample qualitative diagnostic questions for } \\
\text { use in task-oriented projects are listed for } \\
\text { each element/sub-element and are, for the } \\
\text { most part, based on adaptations of items } \\
\text { from the Kitson et al. Appendix related to } \\
\text { Evidence [5]. Their } 2008 \text { Appendix is said to } \\
\text { outline "diagnostic and evaluative } \\
\text { measures," but it is not a formal "tool." }\end{array}$ \\
\hline & $\begin{array}{l}\text { EBP Characteristics = Attributes describing } \\
\text { the nature of the implementable form of } \\
\text { the evidence/practice recommendation. }\end{array}$ & $\begin{array}{l}\text { - Perceptions of key stakeholders can } \\
\text { be influenced by various attributes } \\
{[7,33] \text { related to this EBP and its }} \\
\text { evidentiary source/s. }\end{array}$ & $\begin{array}{l}\text { - Initial, diagnostic evaluation is herein } \\
\text { referenced as the first stage of an } \\
\text { implementation project's formative } \\
\text { evaluation [20]. }\end{array}$ \\
\hline
\end{tabular}

PARIHS = Promoting Action on Research Implementation in Health Services; EBP = evidence-based practice; ORCA = Organizational Readiness to Change Assessment.

the following: "external facilitators were likely to use or integrate other implementation interventions, while performing this problem-solving and supportive role" [19].

The Guide has been disseminated within the VA as a resource for implementation scientists. Individuals familiar to the authors (personal communications) have reported using the modified framework in their studies or intending to put it to use in the near future. Such uses included the following:

- Guiding new investigators looking for "theoretical" assistance

Table 7 Illustration of Guide content: sample material for a sub-element

\begin{tabular}{|c|c|c|c|}
\hline $\begin{array}{l}\text { Related } \\
\text { Sub- } \\
\text { elements }\end{array}$ & Conceptual definitions & $\begin{array}{l}\text { Detailed observations regarding sub- } \\
\text { elements }\end{array}$ & $\begin{array}{l}\text { Sample, optional questions to guide } \\
\text { formative evaluation }\end{array}$ \\
\hline $\begin{array}{l}\text { Leadership } \\
\text { support }\end{array}$ & $\begin{array}{l}\text { Leadership = Individuals in designated } \\
\text { positions "...at any level of the organization } \\
\text { including executive leaders, middle } \\
\text { management, front-line supervisors, and } \\
\text { team leaders, who have a direct or indirect } \\
\text { influence on the implementation" [7] } \\
\text { Leadership Support = Behaviors, } \\
\text { [verbalized] attitudes, and actions of leaders } \\
\text { that reflect readiness or receptivity to a } \\
\text { change [17] }\end{array}$ & $\begin{array}{l}\text { - In general, relevant leaders' "supportive" } \\
\text { actions can be characterized by various types } \\
\text { of managerial behaviors or responsibilities, } \\
\text { within a change/innovation situation such as } \\
\text { EBP, as listed below. These are not directly } \\
\text { taken from the original PARIHS framework } \\
\text { but rather have been adapted based on the } \\
\text { following: a task-oriented view of related } \\
\text { PARIHS sub-elements, supplemental } \\
\text { information from relevant papers [17][36,37], } \\
\text { relevant EBP behaviors of transformational } \\
\text { leaders [17], and an effort to use language } \\
\text { more familiar to targeted researchers. } \\
\text { - Role clarity, e.g., ensuring transparency } \\
\text { regarding both project-related and relevant } \\
\text { change-related role responsibilities and } \\
\text { accountabilities. }\end{array}$ & $\begin{array}{l}\text { - To what extent do leaders show active } \\
\text { and visible support for this change or this } \\
\text { type of EBP and implementation? } \\
\text { o Is the leader willing to engage with the } \\
\text { study team for planning? } \\
\text { o Is the leader willing to provide } \\
\text { connections/entrees for the study team? } \\
\text { o Does the leader have experience/ } \\
\text { comfort in this role? } \\
\text { o Does the leader hold service directors } \\
\text { accountable for collaboration and } \\
\text { coordination in such change efforts/in this } \\
\text { effort? } \\
\text { - To what extent are appropriate } \\
\text { stakeholders or teams held accountable } \\
\text { and incentivized or rewarded to carry out } \\
\text { the implementation? } \\
\text { o What about past experiences with this } \\
\text { type of change? } \\
\text { - To what extent does the leader indicate } \\
\text { the willingness to and in fact does the } \\
\text { leader communicate the priority of this } \\
\text { implementation? }\end{array}$ \\
\hline
\end{tabular}


- Simplifying selection of diagnostic/evaluative questions relevant to a targeted EBP, followed by organization of those questions into a semi-structured interview - Defining specifics of an external facilitation intervention (e.g., the level of interaction and type of external facilitator needed), thus making formative evaluation easier [20]

- Facilitating thinking about what Successful Implementation would look like in a study and how that would be measured

- Assisting in the preparation of a proposal wherein use of a theoretical framework and related design decisions could more clearly be explained to reviewers.

In conclusion, the PARIHS synthesis paper suggested that "the single greatest need for researchers using PARIHS, and other implementation models, is to use the framework prospectively and comprehensively, and evaluate that use relative to its perceived strengths and issues for enhancing successful implementation" [1]. Those using this manuscript to either implement a targeted EBP or study such an implementation thus are encouraged to use the Guide prospectively/comprehensively and to evaluate its use. Formal evaluations and critical reflections regarding the usefulness and limitations of our revised PARIHS and Guide could thereby promote continued evolution of this promising framework.

\section{Additional material}

Additional file 1: EVIDENCE REFERENCE TOOL: Definitions for a “Revised" EVIDENCE Element: EVIDENCE \& EBP CHARACTERISTICS

This Evidence Reference Tool provides explicit definitions for this element and its sub-elements; related, detailed explanations and observations; and sample, optional questions to guide formative evaluation.

Additional file 2: CONTEXT REFERENCE TOOL: Definitions for a "Revised" PARIHS "Context" Element: Contextual Readiness for Targeted EBP Implementation. This Context Reference Tool provides explicit definitions for this element and its sub-elements; related, detailed explanations and observations; and sample, optional questions to guide formative evaluation

Additional file 3: FACILITATION REFERENCE TOOL: Definitions for a "Revised" PARIHS FACILITATION Element. This Facilitation Reference Tool provides explicit definitions for this element and its sub-elements; related, detailed explanations and observations; and sample, optional questions to guide planning the critical details of implementation.

Additional file 4: SUCCESSFUL IMPLEMENTATION TOOL: Definitions for a "Revised" PARIHS Successful Implementation Element. This Successful Implementation Reference Tool provides information on three foci for evaluation of this component of the framework; related definitions and key issues; and observations and suggestions regarding relevant measurements.

\section{Acknowledgements}

This material is based upon work supported by the U.S. Department of Veterans Affairs, Office of Research and Development Health Services R\&D Program.
We wish to acknowledge Linda Mclvor, Diane Hanks, Sarah Krein, and Jacqueline Fickel, as well as members of the original synthesis group [1], for their feedback and input regarding the Guide. We would also like to acknowledge the following individuals for their perceptions regarding the use and potential value of using the revised PARIHS Guide: Marylou Guihan, DiJon Fasoli, and Hildi Hagedorn.

The views expressed in this article are the authors' and do not necessarily reflect the position or policy of the Department of Veterans Affairs.

\section{Author details}

${ }^{1}$ Independent Consultant, Amherst, Massachusetts, USA. ${ }^{2}$ Health Services Department, Boston University School of Public Health, Boston,

Massachusetts, USA. ${ }^{3}$ HSR\&D Center for Clinical Management Research and Diabetes QUERI, VA Ann Arbor Healthcare System, Ann Arbor, Michigan, USA. ${ }^{4}$ Northwest HSR\&D Center of Excellence, VA Puget Sound Healthcare System, Seattle, Washington, USA. ${ }^{5}$ Department of Health Services, University of Washington School of Public Health, Seattle, Washington, USA. ${ }^{6}$ VA Substance Use Disorders Quality Enhancement Research Initiative, Minneapolis VA Medical Center, Minneapolis, Minnesota, USA. ${ }^{7}$ Department of Psychiatry, School of Medicine, University of Minnesota, Minneapolis, Minnesota, USA

\section{Authors' contributions}

CBS conceived the design of a Guide and drafted both the initial manuscript and Guide. All authors contributed to development of its content, reviewed drafts, and provided major input and revisions. All authors approved the final manuscript.

\section{Competing interests}

The authors declare that they have no competing interests.

Received: 22 November 2010 Accepted: 30 August 2011 Published: 30 August 2011

\section{References}

1. Helfrich C, Damschroder L, Hagedorn H, Daggett G, Sahay A, Ritchie M, Damush T, Guihan M, Ullrich P, Stetler C: A critical synthesis of literature on the Promoting Action on Research Implementation in Health Services (PARIHS) framework. Implementation Science 2010, 5(1):82.

2. Rycroft-Malone J, Kitson A, Harvey G, McCormack B, Seers K, Titchen A, Estabrooks $C$ : Ingredients for change: revisiting a conceptual framework. Quality \& Safety in Health Care 2002, 11(2):174-180.

3. Rycroft-Malone J, Harvey G, Seers K, Kitson A, McCormack B, Titchen A: An exploration of the factors that influence the implementation of evidence into practice. J Clin Nurs 2004, 13(8):913-924.

4. Rycroft-Malone J, Seers K, Titchen A, Harvey G, Kitson A, McCormack B: What counts as evidence in evidence-based practice? J Adv Nurs 2004, 47(1):81-90.

5. Kitson A, Rycroft-Malone J, Harvey G, McCormack B, Seers K, Titchen A: Evaluating the successful implementation of evidence into practice using the PARiHS framework: theoretical and practical challenges. Implementation Science 2008, 3(1):1.

6. Grol RPTM, Bosch MC, Hulscher MEJL, Eccles MP, Wensing M: Planning and Studying Improvement in Patient Care: The Use of Theoretical Perspectives. The Milbank Quarterly 2007, 85(1):93-138.

7. Damschroder LJ, Aron DC, Keith RE, Kirsh SR, Alexander JA, Lowery JC: Fostering implementation of health services research findings into practice: a consolidated framework for advancing implementation science. Implement Sci 2009, 4:50.

8. Kitson A, Harvey G, McCormack B: Enabling the implementation of evidence based practice: a conceptual framework. Quality in Health Care 1998, 7(3):149-158

9. Harvey G, Loftus-Hills A, Rycroft-Malone J, Titchen A, Kitson A, McCormack B, Seers K: Getting evidence into practice: the role and function of facilitation. Journal of Advanced Nursing 2002, 37(6):577-588.

10. McCormack B, Kitson A, Harvey G, Rycroft-Malone J, Titchen A, Seers K: Getting evidence into practice: the meaning of 'context'. J Adv Nurs 2002, 38(1):94-104

11. Rycroft-Malone J: Promoting Action on Research Implementation in Health Services (PARIHS). In Models and Frameworks for Implementing 
Evidence-Based Practice: Linking Evidence to Action. Edited by: Rycroft-Malone J, Bucknall T. Oxford: Wiley-Blackwell; 2010:

12. McCormack B, McCarthy G, Wright J, Coffey A: Development and Testing of the Context Assessment Index (CAI). Worldviews on Evidence-Based Nursing 2009, 6(1):27-35.

13. Feussner JR, Kizer KW, Demakis JG: The Quality Enhancement Research Initiative (QUERI): from evidence to action. Med Care 2000, 38(6 Suppl 1): 11-6.

14. Stetler CB, McQueen L, Demakis J, Mittman BS: An organizational framework and strategic implementation for system-level change to enhance research-based practice: QUERI Series. Implement Sci 2008, 3:30.

15. Stetler CB, Mittman BS, Francis J: Overview of the VA Quality Enhancement Research Initiative (QUERI) and QUERI theme articles: QUERI Series. Implement Sci 2008, 3:8.

16. Greenhalgh T, Robert G, Bate P, Kyriakidou O, Macfarlane F, Peacock R: How to Spread Good Ideas: A systematic review of the literature on diffusion, dissemination and sustainability of innovations in health service delivery and organisation. National Co-ordinating Centre for NHS Service Delivery and Organisation Research \& Development (NCCSDO) 2004, 1-424.

17. Stetler C, Ritchie J, Rycroft-Malone J, Schultz A, Charns M: Institutionalizing evidence-based practice: an organizational case study using a model of strategic change. Implementation Science 2009, 4(1):78.

18. Helfrich $C, L i Y-F$, Sharp N, Sales A: Organizational readiness to change assessment (ORCA): Development of an instrument based on the Promoting Action on Research in Health Services (PARIHS) framework. Implementation Science 2009, 4(1):38.

19. Stetler C, Legro M, Rycroft-Malone J, Bowman C, Curran G, Guihan M, Hagedorn $\mathrm{H}$, Pineros $\mathrm{S}$, Wallace $\mathrm{C}$ : Role of "external facilitation" in implementation of research findings: a qualitative evaluation of facilitation experiences in the Veterans Health Administration. Implementation Science 2006, 1(1):23.

20. Stetler CB, Legro MW, Wallace CM, Bowman C, Guihan M, Hagedorn H, Kimmel B, Sharp ND, Smith JL: The role of formative evaluation in implementation research and the QUERI experience. J Gen Intern Med 2006, 21(Suppl 2):S1-8.

21. Hagedorn H, Hogan M, Smith JL, Bowman C, Curran GM, Espadas D, Kimmel B, Kochevar L, Legro MW, Sales AE: Lessons learned about implementing research evidence into clinical practice. Experiences from VA QUERI. J Gen Intern Med 2006, 21(Suppl 2):S21-4.

22. Smith MW, Barnett PG: The role of economics in the QUERI program: QUERI Series. Implement Sci 2008, 3:20.

23. Luck J, Hagigi F, Parker LE, Yano EM, Rubenstein LV, Kirchner JE: A social marketing approach to implementing evidence-based practice in VHA QUERI: the TIDES depression collaborative care model. Implement Sci 2009, 4:64

24. Bowman CC, Sobo EJ, Asch SM, Gifford AL: Measuring persistence of implementation: QUERI Series. Implement Sci 2008, 3:21.

25. Stetler CB: Refinement of the Stetler/Marram model for application of research findings to practice. Nurs Outlook 1994, 42(1):15-25.

26. Stetler CB: Updating the Stetler Model of research utilization to facilitate evidence-based practice. Nurs Outlook 2001, 49(6):272-9.

27. Stetler CB, Corrigan B, Sander-Buscemi K, Burns M: Integration of evidence into practice and the change process: fall prevention program as a model. Outcomes Manag Nurs Pract 1999, 3(3):102-11.

28. Lohr KN, Carey TS: Assessing "best evidence": issues in grading the quality of studies for systematic reviews. Jt Comm J Qual Improv 1999, 25(9):470-9

29. Rogers E: Diffusion of Innovations New York: Free Press; 1983.

30. Grimshaw J, Thomas R, MacLennan G, Fraser C, Ramsay C, Vale L, Whitty P, Eccles M, Matowe L, Shirran L, Wensing M, Dijkstra R, Donaldson C: Effectiveness and efficiency of guideline dissemination and implementation strategies. Health Technol Assess 2004, 8(6):iii-iv, 1-72.

31. Grol $\mathrm{R}$, Wensing $\mathrm{M}$, Eccles $\mathrm{M}$ : The implementation of change in clinical practice Edinburgh: Elsevier Butterworth Heinemann; 2005.

32. NHS Centre for Reviews and Dissemination: Getting evidence into practice. Effective Health Care 1999, 5(1):1-16[http://www.york.ac.uk/inst/crd/ EHC/ehc51.pdf].

33. Rogers EM: Diffusion of Innovations New York, NY: The Free Press; 1995.

34. Ellis I, Howard P, Larson A, Robertson J: From workshop to work practice: An exploration of context and facilitation in the development of evidence-based practice. Worldviews Evid Based Nurs 2005, 2(2):84-93.
35. Bahtsevani C, Willman A, Khalaf A, Östman M: Developing an instrument for evaluating implementation of clinical practice guidelines: a testretest study. Journal of Evaluation in Clinical Practice 2008, 14(5):839-846.

36. Sharp ND, Pineros SL, Hsu C, Starks H, Sales AE: A Qualitative Study to Identify Barriers and Facilitators to Implementation of Pilot Interventions in the Veterans Health Administration (VHA) Northwest Network. Worldviews Evid Based Nurs 2004, 1(2):129-39.

37. Helfrich CD, Weiner BJ, McKinney MM, Minasian L: Determinants of Implementation Effectiveness: Adapting a Framework for Complex Innovations. Med Care Res Rev 2007, 64(3):279-303.

doi:10.1186/1748-5908-6-99

Cite this article as: Stetler et al:: A Guide for applying a revised version of the PARIHS framework for implementation. Implementation Science 2011 6:99.

\section{Submit your next manuscript to BioMed Central and take full advantage of:}

- Convenient online submission

- Thorough peer review

- No space constraints or color figure charges

- Immediate publication on acceptance

- Inclusion in PubMed, CAS, Scopus and Google Scholar

- Research which is freely available for redistribution

Submit your manuscript at www.biomedcentral.com/submit
Biomed Central 